\title{
LA PRÁCTICA REFLEXIVA, UNA PEDAGOGía PARA FORMAR PROFESIONALES
}

Ernesto Sánchez Jerez ${ }^{2}$ Universidad Santo Tomás - Bucaramanga

\section{Resumen}

El texto presenta el proceso de reificación de la ciencia y la manera como el individuo retoma su papel de sujeto agente. Además tiene como referente a Donald Schön, quien aborda problemas del profesional formado desde la racionalidad técnica y propone un cambio de mirada epistemológica de la práctica del mismo. Finalmente, retoma la pedagogía de la práctica reflexiva desde Schön y otros autores.

\section{Palabras clave:}

Reificación de la ciencia, formación profesional, racionalidad técnica, práctica reflexiva, reflexión desde la acción.

\section{THE REFLEXIVE PRACTICE, PEDAGOGIGS TO TRAIN PROFESSIONALS.}

\section{Albstract}

The text describes the science reification process and the individual's way of retaking his role as agent subject. Also Donald Schön is a reference point who enters upon professional's problems trained from technical rationality and proposes a change of point of epistemology view of practice itself. Finally, pedagogy of reflexive practice is retaken from Schön and other authors.

\section{Key words:}

Science reification, professional training, technical rationality, reflexive practice, reflection from action.

1 El presente texto es un avance de la segunda parte del referente teórico del proyecto investigativo denominado: Características de la reflexión de los estudiantes de noveno y décimo de Derecho de la Universidad Santo Tomás de Bucaramanga en las interacciones con sus Asesores y Monitores durante las prácticas de Consultorio Jurídico. El proyecto esta inscrito en la tercera convocatoria de investigación interna USTA.

2 Licenciado en Ciencias de la Educación, Filosofía e Historia de la Universidad la Gran Colombia de Bogotá. Magíster en Investigación y Docencia universitaria de la Universidad Santo Tomás de Bogotá. Asesor de Investigación en Postgrados de la Universidad Santo Tomás y en la Facultad de Educación de la Universidad Cooperativa de Colombia de Bucaramanga. Profesor del Departamento de Humanidades de la Universidad Santo Tomás de Bogotá y actualmente en Bucaramanga. Autor de los cursos Virtuales de Filosofía Institucional y de Cultura Teológica para la USTA. ersanchezj@gmail.com 
La práctica reflexiva contra la reificación de la ciencia

Es indudable la importancia de la acción en el Interaccionismo simbólico de Herbert Blumer. "Los grupos humanos son vistos como constituidos por seres humanos comprometidos en la acción" (Blumer, p.6). La acción está constituida por las actividades que realizan en sus encuentros con los otros y qué acuerdan en sus confrontaciones con los otros; las acciones de los individuos pueden ser individuales, colectivas o representativas de organizaciones. Pero lo claro es que la sociedad existe en la acción ${ }^{3}$.

Las acciones de los otros influyen en las acciones propias, hacen que las propias acciones se acoplen a las de los demás ${ }^{4}$. Pero esto no significa que las acciones de los demás determinen las acciones de un individuo como si éste fuera un ser completamente pasivo. El ser humano es un organismo actuante, un sujeto de sus propias acciones, gracias a su self, al poder que tiene de interactuar consigo mismo. En esta auto-interacción, el sujeto tiene en cuenta muchas cosas para actuar o no actuar, para elaborar su plan de acción, surgidas de sus propias construcciones y de las acciones de los demás, es decir, actúa después de un proceso personal de reflexión constituido por indicaciones e interpretaciones ${ }^{5}$. Estas

3 "The action consists of the multitudinous activities that the individuals perform in their life as they encounter one another and as they deal with the sucesión of situations confronting them. The individuals may act singly, they may act collectively, and they may act on behalf of, or as representatives of, some organization or group of others. The activities belong to the acting individuals and are carried on by them always with regard to the situations in which they have to act. The import of this simple and essentially redundant characterization is that fundamentally human grops or society exists in action and must be seen in terms of action" (Blumer, p.6).

4 "The action of others enter to set what one plans to do, may oppose or prevent such plans, may require a revision of such plans, and may demand a very different set of such plans. One has to fit one's own line of activity in some manner to the actions of others" (Blumer, p.8)

5 "The things taken into account cover Duch matters as his wishes and wants, his objectives, the available means for their interpretaciones utilizan esquemas de definición construidas por el individuo a partir de la reflexión sobre las definiciones de los demás y de su propia experiencia ${ }^{6}$.

Las indicaciones e interpretaciones que el sujeto realiza en su reflexión sobre su acción, no se da solamente para oponerse a las acciones de su sociedad, sino aún para aceptar las actuaciones de la tradición. “La acción repetitiva y estable es un resultado de un proceso interpretativo así como una nueva forma de acción conjunta que es desarrollada por primera vez" (Blumer, p.18). En el caso de un rompimiento con las acciones tradicionales, Blumer realiza una acertada consideración: “la nueva forma de acción conjunta siempre emerge del contexto de una previa acción conjunta y está conectada con él"7. Esta idea es sustentada también por Ausubel, quien sostiene que un aprendizaje es significativo si se relaciona con los conocimientos anteriores de manera intencional ${ }^{8}$.

Sin embargo, la sociedad, por medio de la reificación, trata de imponerse sobre el sujeto actor. Berger y Luckmann analizan este proceso hasta llegar a la

achievement, the actions and anticipated actions of others, his image of himself, and the likely result of a given line of action. His conduct is formed and guided through such a process of indication and interpretation. In this process, given lines of action may be started or stopped, they may be abandoned or postponed, they may be confined to mere planning or to an inner life of reverie, or if initiated, they may be transformed" (Blumer, p.15-16).

6 "The interpretation depends instead on the schemes of definition which the individual possesses and on the nature of his own act in terms of his purposes, aims or directions. His schemes of definitions incorporate the definitions of his fellows, i.e., the expectations of how one should act in a given situation. They may also incorporate schemes of his own as they have been forged in his experience" (Blumer, p.110).

7 "One cannot understand the new form without incorporating knowledge of this continuity into one's analisis of the new form. Joint action not only represents a horizontal linkage, so to speak, of the activities of the participants, but also a vertical linkage with previous joint action" (Blumer, p.20).

8 “En otras palabras, la única manera en que es posible emplear las ideas previamente aprendidas en el procesamiento (internalización) de ideas nuevas consiste en relacionarlas intencionadamente con las primeras. Las ideas nuevas, que se convierten en significativas, expanden también la base de la matriz de aprendizaje" (Ausubel, 1968, p.58, en Novak, 1977, p.70). 
reificación de la ciencia, y plantean la manera de volver a darle al individuo la importancia perdida de agente. Fourez actúa en esta última dirección.

Para Berger y Luckmann, el mundo social pasa por tres momentos: la externalización, la objetivación y la internalización (Berger y Luckmann, p.83).

La externalización se da porque el hombre está abierto al mundo por su falta de instintos. La flexibilidad de su organismo se complementa con una "susceptibilidad frente a la interferencia socialmente determinada" (Berger y Luckmann, p.69). Por eso, la construcción de su actividad indeterminada e inestable y de su mismo yo es realizada en un ambiente social, con la ayuda de los otros ${ }^{9}$. Su misma actividad de observación, está organizada por la sociedad, de la vida cotidiana. Un niño tiene el "sentimiento de realidad" cuando siente que sus padres o familiares ven lo mismo que él (Fourez, p.38). Igual sucede con los científicos ${ }^{10}$ "Así que los científicos no son individuos que observan el mundo a partir de cero; son los participantes de un universo cultural y lingüístico en el que se insertan sus proyectos individuales y colectivos" (Fourez, p.30).

La objetivación incluye cuatro pasos: la habituación, la institucionalización, la legitimación y la objetividad.

Para Berger y Luckmann, "toda actividad humana está sujeta a la habitua-

\footnotetext{
9 "El ser humano no se concibe dentro de una esfera cerrada de interioridad estática; continuamente tiene que externalizarse en actividad. Esta necesidad antropológica se funda en el equipo biológico del hombre. La inestabilidad inherente al organismo humano exige como imperativo que el hombre mismo proporcione un contorno estable a su comportamiento; él mismo debe especializar y dirigir sus impulsos. Estos hechos biológicos sirven como presupuesto necesario para la producción del orden social" (Berger y Luckmann, p.73-75).

10 “Los científicos también suelen tener la impresión de ver 'algo real' cuando están en una comunidad -la comunidad científicaque aprueba su descripción. Por el contrario, cuando tienen la impresión de ser los únicos en observar un fenómeno, tienen un sentimiento de irrealidad e incluso tienden a no creer en sus observaciones" (Fourez, p.39).
}

ción" (p.74). Ésta es una táctica que hace que una actividad útil se pueda repetir sin esfuerzos, en el terreno social y aún en la soledad del individuo que se repite a sí mismo los pasos de su hábito. "En otras palabras, aún el hombre solitario tiene por los menos la compañía de sus procedimientos operativos" (p.74).

Cuando varios actores realizan los mismos hábitos hasta el punto de hacerlos un tipo diferenciado de acciones, tenemos una institución. "La institucionalización aparece cada vez que se da una tipificación recíproca de acciones habitualizadas por tipos de actores. Dicho en otra forma, toda tipificación de esa clase es una institución" (Berger y Luckmann, p.76). La institución identifica a los actores y a las acciones. Por ejemplo, la institución de la biología genética define las acciones que se realizan y al actor que las ejecuta como pertenecientes a esa institución. Algo inherente a la institución es el control que ejerce sobre las acciones de los actores $^{11}$. Se considera que la comunidad científica está formada por individuos que saben observar, captando las cosas "como son sin que intervenga ningún elemento humano; las leyes surgirían de esas observaciones y luego se comprobarían con experiencias que obedecerían a una lógica y una racionalidad únicas y claras" (Fourez, p.69). Esto convierte a los científicos en un tipo superior de humanidad más cerca de la verdad, poseedora de una racionalidad científica eterna. Pero si consideramos a esa comunidad como "vinculada con una forma socialmente reconocida y eficaz de abordar nuestra relación con el mundo" (Ib.), empeza-

11 "Las instituciones, por el hecho mismo de existir, también controlan el comportamiento humano estableciendo pautas definidas de antemano que lo canalizan en una dirección determinada, en oposición a las muchas otras que podrían darse teóricamente. Importa destacar que este carácter controlador es inherente a la institucionalización en cuanto tal, previo o aislado de cualquier mecanismo de sanción establecido específicamente para sostén de una institución" (Berger y Luckmann, p.76). 
mos a ver a los científicos como seres humanos normales con una función social importante ${ }^{12}$.

Para que una institución se imponga y permanezca socialmente activa, es importante legitimarla, esto es, explicarla y justificarla (Berger y Luckmann, p.84). La explica "atribuyendo validez cognoscitiva a sus significados" y la justifica "adjudicando dignidad normativa a sus imperativos prácticos" (p.122). En el caso de las teorías científicas, esta explicación y esta justificación, tal como la vemos, se fundamenta en valores desinteresados y en razones objetivas muy racionales, cuando en su legitimación originaria, muchas veces, han influido conceptos religiosos, filosóficos y otros factores extra-teóricos como el poder político, económico y militar ${ }^{13}$.

Una vez que las instituciones han sido legitimadas socialmente, adquieren "objetividad".

“Esto significa que las instituciones que ahora han cristalizado (...) se experimentan como existentes por encima y más allá de los individuos a quienes 'acaece' encarnarlas en

12 "Ya no nos situamos ante un concepto abstracto de racionalidad científica, sino ante prácticas concretas. La comunidad científica y sus prácticas se convierten entonces en un fenómeno humano como tantos otros. Se pueden estudiar sin darles previamente un estatuto excepcional, considerándolos como un tipo de tecnología intelectual para solucionar problemas. De este modo, entendemos el razonamiento científico como una forma socialmente reconocida y muy eficaz, de resolver nuestra relación con el mundo" (Fourez, p.69).

13 “Por ejemplo, en el razonamiento de Newton había tantos razonamientos filosóficos y teológicos como 'científicos' (pero la distinción entre esos tipos de razonamientos se ha hecho después, la hemos hecho nosotros, mientras que Newton pensaba de un modo global, mezclando lo que nosotros llamamos física y teología)" (Fourez, p.65). "Antiguamente fueron sobre todo los príncipes quienes reconocían a la comunidad científica; más recientemente, los aliados privilegiados de las comunidades científicas fueron, de hecho, lo que el general Eisenhower llamó el complejo militar industrial (...)." (p.70). "Igual que las tecnologías materiales, las tecnologías intelectuales, que son las ciencias, no pueden desarrollarse sin un acuerdo social sobre nomenclaturas y normas (...). A veces, como en el caso del sistema métrico, es una intervención del Estado lo que conduce a determinadas normalizaciones. Entre ambas tecnologías se pueden percibir múltiples presiones, como las de las empresas o las económicas" (p.85). ese momento. En otras palabras, las instituciones se experimentan ahora como si poseyeran una realidad propia, que se presenta al individuo como un hecho externo y coercitivo" (Berger y Luckmann, p.80).

Cualquier desviación de esta institución se considera depravación, enfermedad o ignorancia (p.89).

En las ciencias, esto sucede cuando se llega a la etapa paradigmática. "Cuando una disciplina está 'establecida' se habla de su período paradigmático. Es la época durante la cual tiene su objeto construido de una forma relativamente estable y sus técnicas relativamente claras" (Fourez, p.88). Las ciencias en este momento son muy útiles porque "simplifican mucho lo 'real' para poder estudiarlo y actuar sobre ello" (Fourez, p.88). Sin embargo, padecen de una debilidad pues se despragmatizan y se alejan de la realidad cotidiana ${ }^{14}$. Para medio conectarse con esta realidad, los científicos utilizan una institución intermediaria, el laboratorio: "un laboratorio está estructurado de tal forma que las experiencias que en él se llevan a cabo pueden analizarse mediante conceptos previstos por el paradigma" (Fourez, p.90). Según Edgar Morin, el paradigma reinante en la mayoría de las ciencias, según las líneas metodológicas de René Descartes, es el de la disyunción. Éste comporta dos principios, el de la separación del todo en pequeñas parcelas de realidad y el de la reducción del conocimiento a la

14 “Es muy posible que las formulaciones teóricas abstractas se fragüen en aislamiento casi total de los vastos movimientos de la estructura social, y en esos casos la competencia entre expertos rivales puede producirse en una especie de vacío societal" (Berger y Luckmann, p.153). “En todos estos casos se puede ver a la vez la fuerza y la debilidad de las aproximaciones paradigmáticas. Son fuertes porque sin ellas no llegaríamos a solucionar la mitad de las cuestiones concretas que resuelven nuestras técnicas modernas. Son débiles porque, al separarse cada vez más de la existencia cotidiana, no resuelven a menudo más que los problemas ideados por los especialistas y no los que experimentan las personas a diario" (Fourez, 88). 
suma de las partes y de lo científico a lo cuantificable ${ }^{15}$.

El problema de esta objetividad paradigmática es que la sociedad tiende a reificar las instituciones, como la ciencia, es decir, a tratarlas como una cosa independiente de la humanidad.

"Se puede expresar de otra manera, diciendo que la reificación es la aprehensión de los productos de la actividad humana como si fueran algo distinto de los productos humanos, como hechos de la naturaleza, como resultados de leyes cósmicas, o manifestaciones de la voluntad divina" (Berger y Luckmann, p.116).

Aquí se están extremando los límites de la objetivación, y entonces la ciencia se convierte en algo "no humano y no humanizable" (p.117).

Una vez objetivada o reificada la institución, la sociedad la hace parte de las nuevas generaciones mediante la internalización. "Los significados objetivados de la actividad institucional se conciben como un 'conocimiento' y se transmiten como tales..." (Berger y Luckmann, p.94). Esta transmisión se realiza mediante la socialización primaria y la secundaria: "este mismo cuerpo de conocimiento se transmite a la generación inmediata, se aprende como verdad objetiva en el curso de la socialización y de ese modo se internaliza como realidad subjetiva" (p.90). Es en este momento del proceso en donde cobra importancia la educación ${ }^{16}$.

\footnotetext{
15 “El principio de reducción comporta dos ramas. La primera es la de la reducción del conocimiento del todo al conocimiento aditivo de sus elementos (...). La segunda rama del principio de reducción tiende a limitar lo conocible a lo que es mensurable, cuantificable, formalizable (...). A partir de aquí, la reducción a lo cuantificable condena todo concepto que no se traduzca por una medida. Ahora bien, ni el ser, ni la existencia, ni el sujeto conocedor pueden ser matematizados o formalizados" (Morin, p.116).

16 "La transmisión del significado de una institución se basa en el reconocimiento social de aquélla como solución 'permanente' a un problema 'permanente' de una colectividad dada. Por lo tanto, los actores potenciales de acciones institucionalizadas deben enterarse sistemáticamente de estos significados, lo cual requiere una cierta forma de proceso 'educativo'. Los significados
}

Uno de los principales objetivos de la educación, consiste en la desreificación de las instituciones para tornarlas en lo que son, productos humanos, aunque eso ha sido "un desarrollo comparativamente tardío en la historia y en cualquier biografía individual" (Berger y Luckmann, p.118). Fourez y Morin proporcionan algunas ideas para desreificar la ciencia.

Para Fourez, hay que cambiar el concepto de lo verdadero separándolo de la idea de adecuación entre la realidad y la inteligencia. Hay que reconocer que la verdad depende más de los sujetos que de los objetos. "El criterio de verdad no consistiría en una adecuación con las cosas tal como son, sino una adecuación con lo 'real de nuestra existencia'. Como propone Toulmin, el criterio último de lo verdadero sería la manera en que permite vivir y adaptarse (...)." (Fourez, p.173). Por otra parte, habría que corregir la noción de saber. “El saber no es simplemente el dominio de las cosas: es una construcción de un ámbito común, mediación para un mundo humano" (p.174). Y, por último, ser conscientes de que la ciencia afecta nuestra forma de vivir y, por lo tanto; debe ser negociada: "dejar de creer que la aventura humana puede llevarse a cabo sin la negociación" (p.175).

Para Morin está claro que hay que cambiar el pensamiento de la separación por el de la unión, por el pensamiento complejo ${ }^{17}$. Es necesario, en consecuencia, cambiar los principios de separación y reducción originados en Descartes, por el principio de Pascal: "Dado que todas las cosas son causadas y causantes, ayudadas y ayudantes, mediatas e inmediatas, y

institucionales deben grabarse poderosa e indeleblemente en la conciencia del individuo" (Berger y Luckmann, p.93).

17 "A un pensamiento que aísla y separa hay que sustituirlo por un pensamiento que distinga y una. A un pensamiento disyuntivo y reductor hay que sustituirlo por un pensamiento de lo complejo, en el sentido originario del término complexus: lo que está tejido junto" (Morin, p.117). 
todas se mantienen por un lazo natural e insensible que une las más alejadas y las más distintas, considero imposible conocer las partes sin conocer el todo, igual que conocer el todo sin conocer particularmente las partes" (en Morin, p.117). Esto implica cambiar el pensamiento dándole importancia a la relación del todo con las partes, a la multidimensionalidad, a la unión de los contrarios, a lo diverso y único ${ }^{18}$. También implica introducir un principio que restablezca la importancia del sujeto en la ciencia.

“El principio de la reintroducción del conocedor en todo conocimiento. Este principio opera la restauración del sujeto, y descubre el problema cognitivo central: desde la percepción a la teoría, científica todo conocimiento es una reconstrucción/ traducción por un espíritu/cerebro en una cultura y un tiempo dados" (p.127).

Con las ideas de Blumer sobre el cambio y el juicio crítico, complementamos las ideas de Fourez y Morin. Para Blumer, el cambio ocurre en el individuo cuando éste interpreta las nuevas situaciones que se le presentan. Esta interpretación depende de lo que el sujeto tiene en cuenta y valora en la situación; de los objetos que desecha, del peso que le da a los objetos que tiene en cuenta, de la unión de los objetos en diferentes patrones ${ }^{19}$. Es claro que para Blumer

18 Existe, efectivamente, necesidad de un pensamiento -que capte que el conocimiento de las partes depende del conocimiento del todo y que el conocimiento del todo depende del conocimiento de las partes, -que reconozca y trate los fenómenos multidimensionales en vez de aislar de manera mutiladora cada una de sus dimensiones, -que reconozca y trate las realidades que son a la vez solidarias y conflictivas (como la democracia misma, sistema que se nutre de antagonismos al mismo tiempo que los regula, -que respete lo diverso, al mismo tiempo que reconoce lo único" (Morin, p.117).

19 "I wish to point out that any line of social change, since it involves change un human action, is necessarily mediated by interpretation on the part of the people cauhgt up in the change -the change appears in the form of new situations in which people have to construct new forms of action. Also, in line with has been said previously, interpretations of new situations are no predetermined by coditions antecedent to the situations but depend on what is taken into account and assessed in the actual situations in which los cambios se producen al exponerse a nuevas situaciones y por el tipo de interpretaciones que el sujeto hace. "Como un organismo capaz de autointeracción él forja su acción a través de un proceso de una definición que incluye elección, valoración y decisión" (p.114).

El profesor Donald Schön ofrece algunos elementos conceptuales y operacionales que contribuyen a la desreificación de la ciencia y a la valoración de la práctica como paradigma válido para obtener conocimiento, al tiempo que soluciona problemas vigentes de la formación profesional en la socialización secundaria universitaria.

La legitimación de la ciencia tiene en Bacon y Hobbes, en el siglo XVIII, a dos de sus grandes adalides. Ellos propugnaban "la idea de que el progreso humano se lograría poniendo a la ciencia en disposición de crear tecnología para el logro de fines humanos" (Schön, 1983, p.40). Pero la filosofía que le da el fundamento a través del registro de los triunfos científicos es el positivismo de Augusto Comte, a principios dels. XIX, quien sostuvo que la ciencia empírica es la única forma de conocimiento, que hay que limpiar el mundo del misticismo y que hay que extender la ciencia y la tecnología por todos los ámbitos de la sociedad ${ }^{20}$. Insta-

behavior is formed. Variations in interpretation may readly occur as different acting units cut out different objects in the situation,or give different weightd to the objects which they note, or piece objects together in different patterns. In formulating propositions of social change, it would be wise to recognize that any given line of such change is mediated by acting units interpreting the situations with which they are confronted" (Blumer, 89).

20 "Con este espíritu, en la primera mitad del siglo XIX, Augusto Comte expresó por primera vez las tres doctrinas principales del positivismo. Primero, había la convicción de que la ciencia empírica no era tan sólo una forma de conocimiento, sino la única fuente de conocimiento positivo del mundo. Segundo, había la intención de limpiar la mente humana del misticismo, la superstición y las otras formas de pseudos-conocimientos. Y finalmente, había un programa para extender el conocimiento científico y el control tecnológico por toda la sociedad humana, para hacer la tecnología, como dijo Comte, 'no por más tiempo exclusivamente geométrica, mecánica o química, sino también y primariamente política y 
lado objetivamente el positivismo en la sociedad, el Círculo de Viena, a principios del s. XX, declaraba la verdad de las proposiciones analíticas de la lógica y las matemáticas y de las proposiciones empíricas, ya que "las únicas exposiciones significativas del mundo eran aquellas basadas en la observación empírica, y todos los desacuerdos sobre el mundo podrían ser resueltos, en principio, mediante la referencia a hechos observables" (p.41). Los científicos, como un grupo social especializado, se dedican a confirmar o desconfirmar sus hipótesis en sus laboratorios por medio de experimentos. Pero las situaciones de la vida no cabían en sus modelos experimentales, "la práctica aparecía como una anomalía incomprensible" (p.42).

El positivismo no aceptaba el conocimiento práctico. "No podemos tratarlo fácilmente como una forma de conocimiento descriptivo del mundo, ni podemos reducirlo a los esquemas analíticos de la lógica y las matemáticas" (Schön, 1983, p.42). Los positivistas terminan reduciendo la práctica a un mero instrumento para las finalidades: “El conocimiento práctico había de ser construido como conocimiento de la relación de los medios con los fines" ( $p$. 42). La práctica se reduciría a tratar de encontrar los medios más adecuados para obtener los fines. Si hubiera dudas sobre cuáles medios son los más convenientes, se recurriría al laboratorio, al experimento, enmarcándose dentro de alguna teoría científica ${ }^{21}$.

Esta minusvaloración de la práctica se agranda con el éxito de las instituciones investigativas durante la segunda gran guerra mundial. Estados Unidos da ori-

moral'" (Schön, 1983, p.41).

21 "La cuestión '¿Cómo debería actuar?' podría convertirse en una cuestión científica, y los mejores medios podrían ser seleccionados por el uso de la técnica con base científica" (Schön, 1983, p.42). gen al Office of Scientific Research and Development con la misión de investigar para el conflicto bélico en el campo militar y médico. Esta oficina creará el Proyecto Manhattan, constructor de las primeras bombas atómicas, que tantos triunfos militares le proporcionaron a Estados Unidos ${ }^{22}$. Esto colabora a la reificación del positivismo y al surgimiento de su correspondiente epistemología. "La racionalidad técnica es la epistemología positivista de la práctica" (Schön, 1983, p.39).

Como epistemología, la racionalidad técnica separa los medios de los fines, la investigación de la práctica, el saber del hacer. Para ella, la resolución de un problema consiste en hallar los medios adecuados para lograr el objetivo deseado, la realización de una práctica se reduce a aplicar las teorías y las técnicas descubiertas por la investigación científica, la ejecución de una acción es solo llevar a cabo una decisión técnica. Para ella, la realidad, los hechos, son lo dado, lo objetivo, el marco referencial para resolver las dudas de la práctica ${ }^{23}$. Es innegable, según esto, que son superiores la investigación y la ciencia

22 "La nueva disciplina de investigación de operaciones se impuso a partir de los esfuerzos estadounidenses y británicos para usar la matemática aplicada a la localización de bombas y a la búsqueda submarina. Y el proyecto Manhattan se convirtió en auténtico símbolo del uso exitoso de la tecnología basada en la ciencia para fines nacionales" (Schön, 1983, p.46).

23 "La epistemología positivista de la práctica descansa sobre tres dicotomías. Dada la separación entre los medios y los fines, la solución del problema instrumental puede verse como un procedimiento técnico que puede medirse por su efectividad en lograr un objetivo preestablecido. Dada la separación entre la investigación y la práctica, la práctica rigurosa puede verse como una aplicación a los problemas instrumentales de las teorías y técnicas basadas en la investigación cuya objetividad y generalidad derivan del método del experimento controlado. Dada la separación entre el saber y el hacer, la acción es tan sólo una implementación y una prueba de la decisión técnica" (Schön, 1983, p.152). “La racionalidad técnica descansa en una concepción objetivista de la relación del práctico competente con la realidad que conoce. Desde este punto de vista, los hechos son lo que son y la verdad de las creencias se comprueba con toda exactitud mediante la referencia a éstos. Todos los desacuerdos significativos se pueden resolver, al menos en principio, mediante la referencia a los hechos. Y el conocimiento profesional descansa fundamentalmente sobre una base de hechos" (Schön, 1987, p.44). 
con relación a la práctica. Esto va a influir tanto sobre la universidad como sobre la formación de los profesionales. La racionalidad técnica: "Se institucionalizó en la universidad moderna, fundada al final del siglo XIX, cuando el positivismo estaba en su apogeo, y en las escuelas profesionales, que aseguraron su lugar en la universidad en las primeras décadas del siglo XX" (Schön, 1983, p.40).

En los comienzos del siglo XIX, Alexánder von Humboldt crea la universidad moderna en Alemania, fundamentándola sobre el desarrollo de la ciencia pura positivista. Durante la guerra civil de 1861 a 1865, la juventud norteamericana va a realizar sus estudios superiores en Alemania y con gran optimismo importa la universidad científica. Esto da origen a la universidad Johns Hopkins (1876), consagrada prioritariamente a la investigación científica. Esta institución llega a ser el modelo de las universidades en Norteamérica y el paradigma de racionalidad técnica se implanta en los Estados Unidos. Con tanto éxito, que el sociólogo y economista Thorsten Veblen propugna en The Higher Learning in America (1918) por una Universidad dedicada a la ciencia, separada completamente de los institutos profesionalizantes. Éstos son fuentes de problemas que serán resueltos por la universidades, y ésta, con su investigación, regalan a las segundas conocimiento científico para que los futuros profesionales lo apliquen y pongan a prueba (Schön, 1983, p.43-44).

La propuesta de Veblen de separar la Universidad de la formación profesional no tuvo éxito, pues la sociedad necesitaba de los profesionales y la Universidad debería proporcionar a la comunidad algún conocimiento útil. Así que las profesiones entraron a la nueva Universidad, pagando el precio de tener que aceptar la racionalidad técnica con la separación de investigación y práctica, de tareas científicas y tareas profesionales, siendo superiores las primeras ${ }^{24}$.

La epistemología positivista define la práctica profesional desde la ciencia de una manera peyorativa. “De acuerdo con el modelo de racionalidad técnica (...) la actividad profesional consiste en la resolución de problemas instrumentales que se han hecho rigurosos por la aplicación de la teoría científica y de la técnica" (Schön, 1983, p.31). Nathan Glazer (1974) clasifica las profesiones de acuerdo a su proximidad con la racionalidad científico-técnica. Existen las "principales", como la medicina y el derecho, las ingenierías y las profesiones empresariales. $Y$ se dan las "secundarias", como "el trabajo social, biblioteconomía, educación, teología y urbanismo" (Schön, 1983, p.32). Las principales son más cercanas al conocimiento científico porque sus fines y sus contextos son precisos y estables. Las secundarias, al contrario, se alejan del conocimiento científico porque tienen fines y contextos inestables y ambiguos $^{25}$.

24 Se impuso a las profesiones la epistemología positivista y la división del trabajo de Veblen. "Lo que sirvió para que la tarea de los científicos, con base en la universidad, y de los especialistas fuera crear la teoría fundamental que profesionales y técnicos aplicarían en la práctica. La función de la escuela profesional sería 'la transmisión a sus estudiantes del conocimiento general y sistemático que es la base de la actuación profesional'. Pero esta división del trabajo reflejaba una jerarquía de clases de conocimiento que también constituía una escala de estatus. Aquellos que creaban una nueva teoría se pensaba que eran superiores en estatus a aquellos que la aplicaban, y las escuelas de 'aprendizaje superior' se pensaba que estaban por encima de las de nivel 'inferior'" (Schön, 1983, p.45).

25 “Las profesiones principales están 'disciplinadas por un fin no ambiguo -salud, éxito en un litigio, provecho- que se arraiga en las mentes de los hombres', y operan en contextos institucionales estables. Por lo tanto, están arraigadas en un conocimiento sistemático y fundamental del que el conocimiento científico es el prototipo, o tienen además 'un alto componente de conocimiento estrictamente tecnológico, que se basa en la ciencia, en la educación que proporcionan'. Por el contrario, las profesiones secundarias adolecen de unos fines inestables y ambiguos y de unos contextos o prácticas institucionales inestables y son, por lo tanto, incapaces de desarrollar una base de conocimiento profesional sistemático y científico. Para Glazer, el desarrollo de 
Para la racionalidad técnica tres componentes en el conocimiento profesional: una disciplina subyacente o ciencia básica, una ciencia aplicada o componente de ingeniería y un componente de habilidad y actitud ${ }^{26}$. Estos componentes aparecen en los planes de educación de las profesiones, que son estructurados en ese mismo orden. "La regla es: primero, la ciencia básica pertinente y la ciencia aplicada; después, las destrezas para su aplicación a los problemas del mundo real de la práctica" (Schön, 1983, p.36). El conocimiento verdadero está en las ciencias; las habilidades para usarlo son posteriores y de menor importancia, son "un tipo ambiguo y secundario de conocimiento. Después de todo, hay algo de perturbador en denominarlas 'conocimiento'" (p.37). Este tipo de estructura está clara en el curriculum de medicina: en primer lugar se estudia bioquímica, fisiología, patología y farmacología en los laboratorios; posteriormente se hacen las prácticas clínicas en los hospitales (p.37). Este modelo se generalizó con más o menos fortuna a las demás carreras profesionales.

Las profesiones enraizadas en la racionalidad técnica tenían un futuro lleno de éxitos. Así lo hacía la revista Daedalus en 1963. Expresaba sin ambages: “En todos los ámbitos de la vida norteamericana triunfan las profesiones" (en Schön, 1983, p.17). Daniel Bell anunció en 1972 que los profesionales y técnicos serían el $25 \%$ de la fuerza laboral en el año 2000 (p.18).

la base de un conocimiento científico depende de fines fijos, inequívocos, porque la práctica profesional es una actividad instrumental" (Schön, 1983, p.33).

26 Según Édgar Schein, estos tres componentes son: "1. Una disciplina subyacente o ciencia básica componente sobre la cual descansa la práctica, o partir de la cual se desarrolla. 2. Una ciencia aplicada, o componente de 'ingeniería', de la cual se derivan muchos de los procedimientos de diagnóstico cotidianos y as soluciones a los problemas. 3. Un componente de habilidady actitud que concierne a la real ejecución de los servicios al cliente, utilizando el conocimiento básico y aplicado subyacente" (Citado por Schön, 1983, p.34).
Pero el triunfo de las profesiones orientadas por la racionalidad técnica empezó a ser cuestionado por varios motivos. En primer lugar por carecer de vivencia de valores y en segundo lugar por ser ineficaces. Se les tilda de ser egoístas, de no tener en cuenta los intereses de la sociedad ${ }^{27}$. Se les acusa de no ser eficaces en las soluciones, pues en lugar de resolver los problemas, los aumentan ${ }^{28}$. Se les considera villanos ineficaces ante el aumento de "Ia polución ambiental, la desigualdad y el alto coste de la atención médica" (Schön, 1983, p.21). En tercer lugar, porque las profesiones no otorgan el estatus esperado, sino que han entrado en un descenso económico tal que ya se habla de "proletarización" de las profesiones, y hasta se encuentran profesionales sindicalizados (p.24). Toda la sociedad, por lo anterior, ha entrado en una "crisis de confianza en las profesiones" (Ib.). ¿A qué se debe este fenómeno?

Puede ser porque el poder político de los gobernantes y el poder económico de los empresarios, se imponen sobre la mejor buena voluntad de los profesionales. O quizás puede ser por falencias en el conocimiento de los profesionales: "¿Es el conocimiento profesional adecuado para satisfacer los propósitos adoptados por las profesiones?" (Schön, 1983, p.24). O probablemente, la causa fundamental sea una falta de adecuación entre el conocimiento del profesional y sus prácticas. Los profesionales se enfrentan a situaciones prácticas y no han sido

27 Supuestamente los profesionales contribuyen al bienestar social “Pero tanto la crítica popular como la académica acusa a los profesionales de estar al servicio de sí mismos a expensas de sus clientes, pues ignoran sus obligaciones respecto al servicio público, y fracasan en la vigilancia efectiva de sí mismos" (Schön, 1983, p.22).

28 "La evidencia de la inefectividad profesional se ha presentado en revelaciones académicas y periodísticas des desastres dirigidos profesionalmente -la guerra de Vietnam, la bahía de Cochinos, el accidente nuclear en Three Mile Island, la quiebra de la ciudad de Nueva York, para nombrar tan solo unos cuantos ejemplos de este género-" (p.23). 
formados para hacerlo. "Los profesionales están llamados a realizar tareas para las que no han sido educados y 'el nicho no se adecúa por más tiempo a la educación, o la educación ya no se adecúa al nicho'" (p.25).

La razón estriba en que el conocimiento obtenido en las universidades no se adecúa a las características de la situación práctica: "complejidad, incertidumbre, inestabilidad, carácter único y conflicto de valores" (Schön, 1983, p.25). Las situaciones prácticas son complejas, tejidas por muchos elementos y factores. "En campos tales como la medicina, la gestión y la ingeniería, por ejemplo, los profesionales destacados hablan de la nueva conciencia de una complejidad que se resiste a las habilidades y las técnicas de la pericia tradicional" (p.25). Las situaciones prácticas son inestables, cambian a un ritmo más acelerado que en épocas pasadas, lo que exige del profesional una "adaptabilidad sin precedentes". Harvey Brooks cree que esto trae consigo un gran cambio: "Y las cuatro profesiones -medicina, ingeniería, gestión de empresas y educación- pueden llevar el peso de la responsabilidad para generar y conducir este cambio. Esto plantea al profesional una exigencia de adaptabilidad que no tiene precedente" (en Schön, 1983, p.26). La práctica no plantea problemas claros, inequívocos, sino que plantea situaciones "caracterizadas por la incertidumbre, el desorden y la indeterminación" (p.26). La situación práctica no presenta casos repetidos una y otra vez, sino que está caracterizada "por unos acontecimientos únicos" (p.27). De esto dan testimonio eminentes médicos y experimentados ingenieros. "Los profesionales se encuentran frecuentemente enredados en conflictos de valores, metas, propósitos e intereses" (p.28). Y esto es común en las situaciones prácticas de muchos profesionales ${ }^{29}$.

El modo como debe ser abordad una situación práctica contrasta con la manera como la racionalidad técnica resuelve los problemas profesionales. “Los problemas de elección o decisión son resueltos a través de la selección, con los medios disponibles, del más adecuado para los fines establecidos" (Schön, 1983, p.47). Pero en la realidad del profesional no se dan los problemas bien definidos y bien determinados, sino que lo que se le presenta es una situación problemática confusa, indeterminada, incierta. El profesional es quien debe construir el problema. Para hacerlo debe, en primer lugar, encuadrar dicho problema. El encuadre es "el proceso mediante el cual definimos la decisión que se ha de adoptar, los fines que se han de lograr, los medios que pueden ser elegidos" (Schön, 1983, p.47). O de otra manera: es el proceso con el que limitamos las cosas que atenderemos y el contexto en que lo haremos ${ }^{30}$.

El encuadre no puede hacerse acudiendo a procesos técnicos, en primer lugar porque una situación práctica es única y no se adapta a los estándares de la ciencia aplicada (Schön, 1983, p.48). Por otra parte, en la práctica los fines se presentan "confusos y conflictivos", no delimitados claramente, como lo desea

29 "Los profesores están enfrentados a las presiones por incrementar la eficacia en el contexto de presupuestos concertados, de exigencia para que se atengan rigurosamente a 'enseñar los fundamentos', exhortaciones para que alienten la creatividad. construyan la ciudadanía, ayuden a los estudiantes a examinar sus valores. Los trabajadores en los campos de la asistencia social están también divididos entre un código profesional que aboga por la atención a las personas y una presión burocrática para aumentar la eficacia en el tratamiento de casos" (Schön, 1983, p.28).

30 "Cuando planteamos el problema, seleccionamos lo que trataremos como los 'asuntos' de la situación, establecemos los límites de nuestra atención respecto a ello, le imponemos una coherencia que nos permita decir lo que está equivocado y en qué direcciones necesita ser cambiada la situación. Encuadrar el problema es un proceso en el que, interactivamente, nombramos las cosas a las que prestaremos atención y enmarcamos el contexto en el que las atenderemos" (Schön, 1983, p.48). 
la racionalidad técnica; tampoco aquí son útiles los procesos técnicos sino que hay que acudir al encuadramiento no técnico ${ }^{31}$. El encuadramiento no técnico precede a la habilidad técnica y no al revés (p.49).

En general, los profesionales ubican sus prácticas en dos terrenos: uno ' "elevado y sólido" y otro "bajo y pantanoso". En el primero se hallan las prácticas en "donde los profesionales pueden hacer uso efectivo de la teoría y la técnica basadas en la investigación" (Schön, 1983, p.49). En el segundo, las "situaciones son confusos 'revoltijos' sin posible solución técnica" (Ib.). En el terreno alto se encuentran los problemas rigurosos científicamente, pero no relevantes para la sociedad; en el bajo, los problemas relevantes socialmente pero no tan científicos. El profesional se encuentra en un dilema: "rigor o relevancia" (lb.). Algunos escogen el terreno bajo relevante, otros, el terreno alto riguroso.

Algunos profesionales egresados de la racionalidad técnica ${ }^{32}$ escogen el terreno bajo, el de la relevancia, actuando de tres maneras: escogen los casos menos exigentes y olvidan su formación original, o utilizan los “paradigmas formales como 'sondas'

31 "La racionalidad técnica depende del acuerdo acerca de los fines. Cuando los fines son fijos y claros, entonces la decisión de actuar puede presentarse como un problema instrumental. Pero cuando los fines son confusos y conflictivos, no hay todavía un 'problema' que resolver. Un conflicto de fines no puede ser resuelto mediante el uso de técnicas derivadas de la investigación aplicada. Más bien a través de procesos o técnicas de encuadramiento de la situación problemática podemos organizar y clarificar tanto los fines que se han de lograr como los posibles medios con que hacerlo" (Schön, 1983, p.49).

32 "Desde la perspectiva de la racionalidad técnica, como ya he indicado, un práctico competente se preocupa siempre por los problemas instrumentales. Trata de encontrar los medios más idóneos para la obtención de determinados fines que no admiten ambigüedades: en medicina, la salud; en la ley, el éxito en el pleito; en los negocios, el beneficio. Su eficacia se mide por su éxito en encontrar, en cada caso, las acciones que producen los efectos deseados que son consecuentes con sus objetivos. Vista así, la competencia profesional consiste en la aplicación de teorías y técnicas derivadas de la investigación sistemática, preferiblemente científica, a la solución de problemas instrumentales de la práctica" (Schön, 1987, p.42) o 'metáforas' útiles solamente como fuentes de nuevas perspectivas en las situaciones complejas" (Schön, 1983, p.51). Pero la mayoría de ellos escogen el terreno alto, el del rigor, "reducen la situación práctica para que encaje con el conocimiento profesional" (Ib.). Esta reducción es hecha de tres maneras: no tienen en cuenta los "datos que caen fuera de sus categorías"; utilizan "categorías chatarra", como la personalidad o los políticos, para explicar los datos discrepantes; "o pueden tratar de forzar la situación hacia un paradigma que es apto para el uso de las técnicas disponibles" (lb.). Los profesionales practicantes del terreno alto, "piensan como un...", médico, abogado, ingeniero, que utiliza los conocimientos disponibles para resolver los casos problemáticos. “El práctico competente se concibe, desde esta perspectiva, como alguien que sigue reglas para la recogida de datos, la inferencia y la comprobación de hipótesis, lo que le permite establecer claras conexiones entre las situaciones concretas y el cuerpo de conocimiento profesional, en donde tales conexiones resultan inicialmente problemáticas" (Schön, 1987, p.43). Esta manera de pensar y de actuar, por supuesto, no tiene en cuenta verdaderamente las situaciones prácticas, sobre todo cuando éstas divergen de las técnicas disponibles.

Consideremos ahora la práctica partiendo de una epistemología de la práctica reflexiva o de la reflexión desde la acción. Para ésta, "Ios medios y los fines están estructurados de forma interdependiente", "la práctica es un tipo de investigación", y "el saber y el hacer son inseparables" (Schön, 1983, p.152). Los problemas y los fines no se encuentran dados, sino que hay que construirlos a partir de una situación problemática problema ${ }^{33}$. Esto lo cono-

33 "Estos investigadores se encuentran con una situación problemática cuya realidad deben construir ellos. Al reformular 
cen los profesionales que reflexionan desde su acción.

Analicemos ahora los conocimientos en la acción. Al hacer algo, por ejemplo, engarajar en reversa una tractomula, el conductor utiliza conocimientos y habilidades, un saber, que él mismo no puede explicar. Y si lo hace, no lo hace de manera apropiada. Estos son los saberes tácitos. "Nuestro conocimiento es de ordinario tácito, está implícito en nuestros patrones de acción y en nuestra sensación respecto a las cosas con las que tratamos. Parece correcto decir que nuestro conocimiento se da desde nuestra acción" (Schön, 1983, p.55). El profesional usa en su actividad propia muchos saberes tácitos que no pueden ser descritos con exactitud, como ciertos fenómenos observados, los criterios utilizados para emitir juicios, las reglas que aplica ${ }^{34}$. Es tácito el saber cómo del goleador de las grandes ligas, del contador que en segundos descubre "hechos significativos" en un estado de cuentas. Son tácitos los rasgos para el reconocimiento de las caras de los amigos, y son tácitas las reglas para conocer sus estados de ánimo. Son tácitos los criterios para conocer si un tejido manual está bien elaborado (p.56-59). Este saber tácito es espontáneo, inaprendido e indecible ${ }^{35}$.

el problema de la situación, ellos determinan los rasgos a los que atenderán, el orden que intentarán imponer a la situación, las direcciones en las que tratarán de derivar. En este proceso, identifican tanto los fines que deben buscarse como los medios que han de ser empleados" (Schön, 1983, p.152).

34 "En su práctica diaria hace innumerables juicios de calidad para los que no puede establecer unos criterios adecuados, y exhibe habilidades de las que no puede fijar las reglas y los procedimientos. Incluso cuando hace un uso consciente de teorías basadas en la investigación y de las técnicas, depende de reconocimientos, juicios y hábiles actuaciones que son tácitos" (Schön, 1983, p.56).

35 “En ejemplos como éstos, el saber tiene las siguientes propiedades: -Hay acciones, reconocimientos y juicios que sabemos cómo llevar a cabo espontáneamente; no tenemos que pensar sobre ello previamente o durante su ejecución. -A menudo no somos conscientes de haber aprendido a hacer estas cosas; simplemente nos encontramos haciéndolas. -En algunos casos, fuimos conscientes una vez de las comprensiones que más tarde fueron internalizadas en nuestra sensación de la acción misma. En otros casos podemos no haber sido nunca conscientes de ellas.
En algunas ocasiones reflexionamos desde nuestra acción, "pensamos en lo que estamos haciendo". Esto lo hace el goleador cuando no encuentra cómo controlar a una defensa, los músicos de jazz "cuando improvisan juntos". El pensar sobre lo que se hace ocurre ante lo inesperado, "cuando la actuación intuitiva nos lleva a sorpresas, agradables o prometedoras, o no deseadas, podemos responder con la reflexión desde la acción" (Schön, 1983, p.61). “En tales procesos, la reflexión tiende a centrarse interactivamente en los resultados de la acción, la acción misma, y el saber intuitivo implícito en la acción" (p.62).

Los procesos reflexivos se dan con frecuencia en la práctica profesional. La práctica profesional, por ser la palabra "práctica" ambigua, puede referirse a lo que hace el profesional, a su preparación o a la experiencia por repetición. “En el primer sentido, 'práctica' se refiere a la actuación en una variedad de situaciones profesionales. En el segundo, se refiere a la preparación para la ejecución. Pero la práctica profesional también incluye un elemento de repetición. Un profesional es un especialista que tiene en cuenta ciertos tipos de situaciones una y otra vez" (Schön, 1983, p. 65).

Los expertos hablan en sus prácticas profesionales de "casos". Estos "denotan las unidades con la que se constituye una práctica, y denotan tipos de ejemplos de parecido familiar" (Schön, 1983, p.65). Así, se puede hablar de casos de gastralgia, casos de hurto agravado (...). El profesional acumula experiencia para tratar los casos, es decir, ya no hay sorpresa ante dichos casos. "Su saber desde la práctica tiende a hacerse cada vez más tácito, espontáneo y automático, confirién-

No obstante, en ambos casos, normalmente somos incapaces de describir el saber que nuestra acción revela" (Schön, 1983, p.60). 
dole de ese modo a él y a sus clientes los beneficios de la especialización" (p.65-66). La práctica profesional se hace "repetitiva y rutinaria", y ya no se piensa al actuar. Se ha llegado a la etapa denominada "sobre-aprendizaje". Y es aquí en donde necesita la reflexión que supere la rutina al criticar el saber tácito y al dar un nuevo sentido a las situaciones únicas e inciertas ${ }^{36}$.

Desde la práctica, un profesional puede reflexionar sobre su saber en pasado o en presente, sobre la práctica pasada o sobre la que está realizando. Lo puede hacer rápidamente o muy pausadamente (Schön, p.66), por ejemplo, un abogado puede reflexionar rápidamente en una conciliación o pausadamente para determinar el tipo de demanda que va a realizar. Puede reflexionar sobre distintos elementos de su práctica: normas, estrategias, teorías, sentimientos, encuadre, su propio papel ${ }^{37}$.

Reflexionar desde la acción es "central" para resolver situaciones "divergentes". Cuando el problema no se ajusta a las "categorías ordinarias", la reflexión se enfoca a "criticar su inicial comprensión" y a construir "una nueva descripción de él" y a probarla "mediante un experimento" (Schön, 1983, p.67). Cuando parece inmanejable, la reflexión acude a la construcción de "un nuevo modo de establecer el problema -un nuevo marco que, en lo que llamaré un 'experimento de encuadre', trata de imponer a la situación” (lb.). Cuando el profesional debe dar solu-

\footnotetext{
36 “La reflexión de un profesional puede servir como correctivo del sobre-aprendizaje. A través de la reflexión puede hacer emerger y criticar las comprensiones tácitas que han madurado en torno a las experiencias repetitivas de una práctica especializada, y puede dar un nuevo sentido a las situaciones de incertidumbre o únicas que él puede permitirse experimentar" (Schön, 1083, p.66).

37 "Puede reflexionar sobre las normas y apreciaciones tácitas que subyacen en un juicio, o sobre las estrategias y teorías implícitas en un modelo de conducta. Puede reflexionar sobre los sentimientos respecto a una situación que le ha llevado a adoptar un curso particular de acción, sobre la manera con la que ha encuadrado el problema que trata de resolver, o sobre el papel que ha construido para sí mismo dentro de un contexto institucional más amplio (Schön, 1983, p.67).
}

ciones que parecen incompatibles con otros valores, puede reflexionar sobre la manera en que se ha establecido el problema o su propio papel, para poder "encontrar una vía de integración o elección entre los valores que están en juego en la situación" (p. 67-68).

El profesional, al reflexionar sobre su acción, es un investigador que "construye una nueva teoría de un caso único". Y aquí no separa, sino que une, conjunciona:

No mantiene separados los medios de los fines, sino que los define interactivamente como marcos de una situación problemática. No separa el pensamiento del hacer, racionalizando su camino hacia una decisión que más tarde debe convertir en acción. Dado que su experimentación es un tipo de acción, la implementación está construida dentro de su investigación en curso (Schön, 1983, p.72).

De esta manera, la reflexión desde la acción también puede ser rigurosa, como la investigación científica, y así "el dilema del rigor o la relevancia puede ser resuelto" (p.73).

\section{Pedagogía de la reflexión desde la acción}

Luis Not cuestiona el aprendizaje a partir de la experiencia. En primer lugar expresa que la experiencia se puede referir a dos operaciones distintas, que tienen en común el

"juzgar la validez de un ensayo mediante los hechos que provoca". "La primera se atiene a los hechos escuetos que aparecen cuando se intenta este ensayo pero sin aplicar ningún tratamiento racional, o método a la situación. Engendra ciertamente una inferencia pero sin analizar las razones de su conclusión" (Not, 1979, p.186). “La 
segunda procede de un verdadero razonamiento, en el que los vínculos de antecedente a consecuente se analizan y critican minuciosamente; no se limita a comprobar los estados y resultados, se trata de captar las variaciones que conducen del estado al resultado" (Ib.).

La primera constituye el llamado "empirismo", con el método de ensayo y error que "ajusta la conducta a los efectos producidos", y la segunda, el "método experimental", que se caracteriza por la reflexión y es superior a la primera (lb.). En la primera impera el hábito, el instinto; en la segunda, la inteligencia. La primera se da sin ninguna planificación, al contrario de la segunda que sí es conscientemente planificada ${ }^{38}$.

Not considera que el primer tipo de experiencia se da desde los dos años de edad39, mientras que la experiencia reflexiva, el método experimental, sólo se da a los doce años (Not, 1979, p.187). Esto último porque la reflexión sobre la experiencia necesita poseer la capacidad de establecer "relaciones de orden espacial, temporal, causal" (p.188) y de razonar por medio de "enunciados verbales", de usar la "combinatoria" y de sistematizar la sensibilidad (Ib.)40.

Not hace varias críticas a la experiencia:

38 "En el tanteo, el sujeto actúa sin tener ninguna idea del objetivo y espera hasta que el ensayo tenga éxito; en la experiencia reflexiva, por el contrario, el sujeto instituye la investigación en función de la solución que espera obtener y es ésta la que proporciona la hipótesis" (Not, 1979, p.187).

39 "La primera, desde el segundo año de vida, es la entrada en juego de la representación y el poder de considerar lo virtual, permitiendo sustituir con la experiencia mental la experiencia real; es entonces cuando llega a ser posible la interiorización del tanteo empírico (...)" (Not, 1979, p.188).

40 “Por último el acceso a las operaciones experimentales supone, como lo han demostrado bien B. Inhelder y J. Piaget, el poder de razonamiento en simples enunciados verbales admitidos como hipótesis (lo cual requiere una abstracción de segundo grado), la entrada en juego de coordinaciones nuevas referentes a la combinatoria, por una parte y la sistematización de la sensibilidad por otra parte" (Not, 1979, p.188)
- "Toda experiencia existencial es una prueba más en el sentido afectivo que en el intelectual o en el cognoscitivo" (Not, 1979, p.189). Porque el sujeto se involucra afectivamente en ella.

- "Las informaciones obtenidas de cada experiencia son globales y sincréticas; conciernen a varios sectores del saber que corresponden a otras tantas disciplinas diversas, cada una de las cuales exige un aprendizaje especial (...)" (p.191). Es difícil sistematizarlas y operacionalizarlas.

- “La generalización de lo particular, la racionalización de lo concreto, exigen procesos que cada vez dejan menos lugar a la experiencia efectiva y que cada vez conceden más lugar a otras fuentes o a otros modos de conocimiento" (p.191). Hay que recurrir al conocimiento abstracto y sistematizado.

- Existen "los riesgos de quedarse en lo cotidiano, en la rutina, en el hic et nunc" (p.196). Porque se adquiere un conocimiento demasiado concreto y específico.

- "Por otra parte, cuando la adaptación al mundo está en juego todo error puede llegar a ser fatal y el temor a equivocarse limita las iniciativas" (p.197). Las situaciones reales son inquietantes y perturbadoras.

- Si se arreglan las experiencias hay un peligro: "el de falsear las perspectivas y con ello hacer inoperante la educación, precisamente por estos arreglos que estaban destinados a conferirle eficacia" (p.197).

Esto parece desvirtuar una enseñanza basada en la práctica y la experiencia. Veamos ahora lo que dice Schön acerca de la pedagogía de la práctica del profesional, y cómo resuelve las objeciones de Not. 
En primera instancia, para Schön un profesional es, citando a Evert Hughes (1959), "alguien que recurre al conocimiento extraordinario en asuntos de gran importancia humana" (Schön, 1987, p.41). Para retribuir ese esfuerzo de obtener un gran conocimiento en pro de la sociedad, ésta le otorga garantías para ejercer su profesión, autonomía y cierto monopolio ${ }^{41}$. El profesional puede entonces desempeñar con corrección social su propia profesión, que consiste en "la competencia de una comunidad de prácticos que comparten, en palabras de John Dewey, las tradiciones de una profesión" (Schön, 1987, 41). Las tradiciones de la profesión se concretan en modos de hablar específicos, medios, modos de actuar, sitios propios ${ }^{42}$.

El profesor Schön investiga la práctica de diversos profesionales, en su actividad o en el contexto educativo. Aquí hacemos alusión a las conclusiones que saca después de analizar la asesoría educativa de un arquitecto a una estudiante que diseña una escuela en un terreno inclinado, y la supervisión de un psicoanalista a un residente de psiquiatría que atiende a una paciente (Schön, 1983, p.79-121). Sin ocultar las diferencias, Schön encuentra similitudes en las actuaciones de los dos profesionales: “En ambos ejemplos, el profesional plantea el problema prác-

\footnotetext{
41 Schön sigue exponiendo el pensamiento de Hughes. "El vio la demanda de conocimiento extraordinario del profesional estrechamente relacionada con un pacto paradigmático con la sociedad. En recompensa por el acceso a su conocimiento especial, al profesional se le otorga un mandato especial para el control social en asuntos propios de su pericia, una licencia para determinar quién entrará a formar parte de su campo profesional y un grado relativamente elevado de autonomía en la reglamentación de su práctica" (1987, p.41).

42 "Comparten convenciones de acción que incluyen medios, lenguajes e instrumentos distintivos. Funcionan en el marco de instituciones de un tipo muy particular: por ejemplo, los juzgados, las instituciones educativas, los hospitales y las empresas. Sus prácticas se estructuran en términos de formas particulares de unidades de actividad (por ejemplo: casos, visitas de pacientes 0 lecciones) y son modeladas a nivel social e institucional de manera que se repiten determinados tipos de situaciones" (Schön, 1987,
} p.41-42). tico como un caso único" (p.123). “En ningún ejemplo está dado el problema" (p.124). En ambos casos "hay muchas perspectivas en competencia sobre la naturaleza de la práctica" (Ib.) ${ }^{43}$. Y, por último, "el profesional ofrece una actuación artística" (Ib.) ${ }^{44}$.

Estos son los principales eventos de estas prácticas profesionales, según Schön.

a. El practicante encuentra el problema insoluble: "ha establecido y ha tratado de resolver el problema tal y como lo ha planteado" (Schön, 1983, p.125), pero no lo ha logrado.

b. El problema es replanteado por el profesional y se critica el encuadre del mismo. "Él hace esto implícitamente, dejando que su crítica del viejo problema sea inferido a partir de su modo de reestructurarlo" (lb.).

c. "Al reformular el profesional el problema del estudiante sugiere una dirección para reorganizar la situación(...) El profesional solicita al estudiante que ponga los pies dentro de la situación, que se haga parte de la misma (lb.).

d. El profesional, "toma el problema reestructurado, dirige un experimento tendente a descubrir qué consecuencias e implicaciones pueden hacerse a partir de él" (Ib.).

e. El profesional "trata de adaptar la situación al marco. Esto lo hace a través de una red de acciones,

43 "Hay una controversia no sólo acerca del modo mejor de solucionar problemas específicos, sino acerca de qué problema merece la pena resolver y qué papel debería desempeñar el profesional en la solución" (Schön, 1983, p.124).

44 "La maestría es evidente en su manejo selectivo de grandes cantidades de información, su habilidad para alargar líneas de invención e inferencia, y en su capacidad para mantener varios modos de considerar las cosas al mismo tiempo sin interrumpir el flujo de la investigación. A mí me parece que su arte es, en una medida considerable, un tipo de reflexión desde la acción" (Schön, 1983, p.124). 
consecuencias descubiertas, implicaciones, apreciaciones, y acciones posteriores" (Ib.).

f. Las acciones del profesional "producen cambios inintencionados que proporcionan nuevos significados a las situaciones. La situación replica, el profesional escucha, ya al apreciar lo que está oyendo reestructura la situación una vez más" (p.126).

g. El profesional trata por otros medios de resolver el problema reestructurado, lo que "produce nuevos descubrimientos que requieren una nueva reflexión desde la acción" (lb.).

Brevemente, el proceso se da en una espiral de tres etapas: Apreciación, Acción y Reapreciación (lb.).

Lo que el profesional ha realizado en su práctica reflexiva se denomina "diseño". "Consideraré el diseño como una conversación con los datos de la situación" (Schön, 1983, p.81). Es una conversación creativa: "yo entiendo el diseño como una forma de creación" (Schön, 1987, p.49). Un diseñador es aquél que elabora un producto, o lo representa para que otros lo hagan, en situaciones y con materiales específicos, usando un lenguaje especial ${ }^{45}$. En realidad, todo profesional práctico es un diseñador. "Los abogados construyen casos, argumentos, acuerdos y obras legislativas. Los médicos construyen diagnósticos y regímenes de comprobación y tratamiento. Los

45 “Un diseñador hace cosas. Algunas veces fabrica el producto final; más a menudo realiza la representación -un plano, un programa, 0 imagen- de un artefacto que será construido por otros. Trabaja en situaciones particulares, utiliza materiales específicos, y emplea un medio y un lenguaje característicos. Típicamente, su proceso de fabricación es complejo. Hay más variables -tipos de acciones posibles, normas, e interrelaciones de éstas- que pueden representarse en un modelo finito" (Schön, 1983, p.81). Pero la definición que más le gusta a Schön es la de que el diseñador es aquél que determina las situaciones. "Sin ignorar ninguna de estas características, me inclino por la visión que Dewey da del diseñador como alguien que transforma situaciones indeterminadas en determinadas" (Schön, 1987, p.50). planificadores construyen planos de un espacio, normas, disposiciones reglamentarias y sistemas para armonizar intereses en litigio..." (Schön, 1987, p.50). La conversación del diseñador con la situación es una reflexión sobre la misma. "En la respuesta a las réplicas de la situación, el diseñador reflexiona desde la acción a propósito de la construcción del problema, las estrategias para la acción, o el paradigma de fenómenos que han estado implícitos en cada uno de sus pasos" (Schön, 1983, p.81).

Donald Schön es taxativo: a diseñar se aprende, no se enseña. Porque el diseño posee estas características:

1. “Un proceso de diseño competente es una forma de conocimiento en la acción" (Schön, 1987, p.146). El diseñador conoce ciertas reglas; y al aplicarlas no utiliza el ensayo y error sino la experimentación que considera "la apreciación de los resultados de los pasos anteriores. La aplicación de este tipo de regla a un caso concreto debe estar mediada por una forma de reflexión en la acción" (lb.).

2. "Diseñar es una habilidad integral" (Ib.). Porque el diseño se presenta como una totalidad en donde todas las piezas están interrelacionadas. No se puede aprender por partes, pues se desbarata el sentido del todo.

3. “Un proceso competente de diseño depende de la habilidad del diseñador para reconocer y apreciar cualidades del diseño deseables o no deseables" (p.147). Y esta habilidad solo se aprende a través de la práctica y la experimentación práctica.

4. “Lo que es cierto para la descripción y el reconocimiento de las cualidades del diseño lo es, también, en 
términos generales, para la descripción y el reconocimiento de un diseño competente" (p.148). Esto se obtiene mediante una reflexión continua sobre la práctica.

5. "Diseñar es una actividad creadora" (p.149). La reflexión sobre la situación genera nuevos significados, nuevas rutas. $Y$ esto se produce practicando, en la inmersión en la acción reflexiva, porque se trata de que el sujeto "vea él mismo" lo nuevo, pues si alguien se lo describe antes ya no es nuevo.

El doctor Schön afirma que si no se puede enseñar el diseño, sí se puede aprender y tutorializar. Según él, hay tres maneras de aprender: por sí mismo, lanzándose a la práctica sólo; convirtiéndose en aprendiz de un práctico experimentado; o por medio de un "prácticum". Un prácticum es una situación fabricada para el aprendizaje, que consiste en la participación en un proyecto que no es real, pero que está relacionado con la realidad, alejado de "Ias presiones, las distracciones y los riesgos que se dan en el mundo real" (Schön, 1987, p.45-46) ${ }^{46}$. El estudiante aprende conscientemente al hacer, e inconscientemente a través de la experiencia y en sus interacciones con los tutores y estudiantes. "El trabajo del prácticum se realza por medio de algún tipo de combinación del aprender haciendo de los alumnos, de sus interacciones con los tutores y los otros

\footnotetext{
46 “Un prácticum es una situación pensada y dispuesta para la tarea de aprender una práctica. En un contexto que se aproxima al mundo de la práctica, los estudiantes aprenden haciendo, aunque su hacer a menudo se quede corto en relación con el trabajo propio del mundo real. Aprender haciéndose cargo de proyectos que simulan y simplifican la práctica, o llevar a cabo, relativamente libre de las presiones, las distracciones y los riesgos que se dan en el mundo real al que, no obstante, el prácticum hace referencia. Se sitúa en una posición intermedia entre el mundo de la práctica, el mundo de la vida ordinaria, y el mundo esotérico de la Universidad. Es también un mundo colectivo por derecho propio, con su propia mezcla de materiales, instrumentos, lenguajes y valoraciones. Incluye formas particulares de ver, pensar y hacer que, en el tiempo y en la medida que le preocupe al estudiante, tienden a imponerse con creciente autoridad" (Schön, 1987, p.45-46).
}

compañeros y de un proceso más difuso de 'aprendizaje experiencial'" (p.46).

Para Schön los "prácticum" se clasifican en tres categorías.

1. Los que preparan técnicamente a los estudiantes enseñándoles a aplicar reglas y procedimientos para solucionar problemas instrumentales. El tutor retroalimenta a los alumnos señalándoles los errores y dándoles la respuesta correcta (Schön, 1987, p.47).

2. Los que enseñan a 'pensar como un/una...' abogado, ingeniero, economista, enseñándoles "hechos y operaciones relevantes" y "formas de indagación" usadas por los profesionales expertos para seguir razonadamente el camino adecuado en situaciones complejas en donde es difícil "clarificar las conexiones entre el conocimiento general y los casos particulares" (p.47). El tutor hará énfasis en las "reglas de indagación" o en "la reflexión en la acción", posibilitando a veces, de esta última manera, la creación de "nuevas reglas y métodos propios" (p.47).

3. Los que enseñan a reflexionar en la acción, generando una reflexión “que va más allá de las reglas existentes, no sólo por el establecimiento de nuevos métodos de razonamiento, como los arriba señalados, sino también por la construcción y comprobación de nuevas categorías de conocimiento, estrategias de acción y maneras de formular los problemas. Los tutores darán énfasis a las zonas indeterminadas de la práctica y a las conversaciones reflexivas con los componentes de la situación" (p.47). Los momentos de la reflexión en la acción son: respuestas espontáneas, resultados inesperados, reflexión, crítica a las 
suposiciones, experimentación falseadora o verificadora ${ }^{47}$.

El tercer prácticum no excluye los dos anteriores, en palabras de Schön:

Quizás aprendamos a reflexionar en la acción aprendiendo a reconocer y aplicar reglas, hechos y operaciones estándar; luego a razonar sobre los casos problemáticos a partir de las reglas generales propias de la profesión; y sólo después llegamos a desarrollar y comprobar nuevas formas de conocimiento y acción allí donde fracasan las categorías y las formas familiares de pensar (Schön, 1987, p.47-48).

Hay varias condiciones que deben ser tenidas en cuenta para realizar un prácticum: el diálogo entre el tutor y el alumno, y las condiciones afectivas.

Entre el estudiante y el tutor se da un diálogo no solo con palabras sino también con acciones, convirtiéndose en una "reflexión en la acción recíproca". "El alumno reflexiona acerca de lo que oye decir al tutor o lo que le ve hacer, y reflexiona también sobre el conocimiento en la acción de su propia ejecución. Y el tutor, a su vez, se pregunta lo que este estudiante revela en

47 Los momentos de la reflexión en la acción son:

- $\quad$ Para empezar, existe aquella situación de la acción a la que traemos respuestas espontáneas y rutinarias (...).

- Las respuestas rutinarias producen una sorpresa, un resultado inesperado, agradable o desagradable, que no corresponde a las categorías de nuestro conocimiento en la acción (...).

- La sorpresa conduce a una reflexión dentro de una acciónpresente. La reflexión, al menos en alguna medida, resulta consciente, aunque no se produzca necesariamente por medio de palabras (...).

- $\quad$ La reflexión en la acción posee una función crítica, y pone en cuestión la estructura de suposición del conocimiento en la acción (...).

- La reflexión da lugar a la experimentación in situ. Ideamos y probamos nuevas acciones que pretenden explorar los fenómenos recién observados, verificar nuestra comprensión provisional de los mismos, o afirmar los pasos que hemos seguido para hacer que las cosas vayan mejor" (Schön, 1987, p.38). cuanto a conocimientos, ignorancia o dificultades y piensa en las respuestas más apropiadas para ayudarle mejor" (Schön, 1987, p.150). Para el estudiante, como para el tutor, se dan dos fases en la práctica: "el arte sustantivo de diseñar que trata de aprender y la reflexión en la acción mediante la cual trata de aprenderlo" (p.151). El tutor tiene entonces tres tareas fundamentales: realiza el proceso de la reflexión desde la acción "afrontando los problemas sustantivos de una tarea propia del diseño, acomodando sus movimientos al tipo de alumno que tiene ante él y construyendo una relación conducente al aprendizaje" (p.154).

Al considerar las condiciones afectivas de un prácticum, éstas son delicadas por parte del estudiante. Éste se lanza al agua sin saber nadar y ello le produce un sentimiento "de pérdida": "Ios estudiantes experimentan una pérdida de control, de aptitud y de confianza; y con estas pérdidas aparecen las sensaciones de vulnerabilidad y dependencia forzada. Bajo estas circunstancias, es fácil ponerse a la defensiva" (p.153). El tutor debe manejar con comprensión y paciencia esta situación afectiva, y resolver con inteligencia el dilema de dejar que el estudiante vaya construyendo su propio aprendizaje a su propio ritmo o soltarle de una toda la teoría implícita en la práctica ${ }^{48}$.

Los problemas afectivos se sortean adecuadamente si el tutor pasa del Ilamado Modelo I de comportamiento interpersonal al Modelo II. El Modelo I parte de los siguientes principios: "consigue el objetivo tal y como yo lo entiendo", "lucha para ganar y evita perder", "evita los sentimientos negativos", y "sé racional" (Schön, 1987,

48 “La versión del dilema del aprendizaje en el caso del tutor opera a dos niveles. Debe aceptar el hecho de que no puede decir a los alumnos sobre el diseño nada que los lleve a comprender ya desde el principio, y luego debe enfrentarse a sus reacciones ante el dilema en el que él ha ayudado a instalarlos" (Schön, 1987, p.153). 
p.226). En este modelo se fomentan las relaciones defensivas y se limita la libertad de acción y el compromiso interno. En él se da el aprendizaje de una sola vuelta: "el aprendizaje de las estrategias o tácticas para conseguir los objetivos personales" (p.226). En cambio, el Modelo II sigue estos principios: "intercambiar información válida", realizar elecciones libres e informadas, hacer un "compromiso interno para la elección y control constante de la puesta en práctica (p.226, 228). Aquí se propician relaciones personales mínimamente defensivas y una alta libertad de elección. En él se da el aprendizaje de doble vuelta en el que también se da "el aprendizaje de los principios rectores que subyacen a las estrategias de comportamiento" (p.229).

Existe el peligro de que el prácticum se aísle de la realidad y de la ciencia, constituyéndose en una isla separada ${ }^{49}$. Para que esto no suceda, debe unir la práctica y la reflexión en la acción con las teorías científicas y las técnicas surgidas de la ciencia. De esta manera, el prácticum resuelve el problema de la formación profesional en las universidades, que muchas veces separa la investigación científica de la práctica. Schön da varios ejemplos de cómo un prácticum puede unir investigación científica y práctica.

En una licenciatura para profesores, cuyos estudiantes dan clases reales como parte de la formación, un prácticum sería de esta manera:

- En una primera instancia, los estudiantes tratarán de comprometerse

49 "Si quiere evitar este destino, debe cultivar actividades que conecten el conocimiento en la acción y la reflexión en la acción de los prácticos competentes con las teorías y las técnicas transmitidas como conocimiento profesional en las disciplinas académicas. Una actividad de este tipo es una forma de investigación que estudia los procesos por los que los individuos adquieren (o no logran adquirir) el arte de la práctica y los procesos que hacen que la tutorización sea más o menos eficaz" (Schön 1987, p.272). "en tareas en las que pueden explorar su propio aprendizaje" (Schön, 1987, p.281). Al realizarlas

"podrían reflexionar sobre sus propios procesos de indagación, examinar sus propios cambios de conocimientos y comparar sus experiencias reales de aprendizaje con las teorías formales del aprendizaje construidas de acuerdo con las normas de la pedagogía. Se les podría ayudar en este proceso exponiéndoles, más adelante, a experimentos y a teorías que tienen que ver con el desarrollo cognitivo" (p.281).

- En una segunda etapa, los estudiantes trabajan sobre las clases en las que interrelacionan con los estudiantes. Comparan el aprendizaje de los niños con el propio. Su enseñanza se transformará en "un proceso de experimentación reflexiva" en el que tratan de descubrir el sentido que le dan los niños a sus actuaciones, es decir, lo que ellos piensan sobre sus propias acciones, sobre sus propias preguntas y respuestas (p.281-282). Reflexionarán sobre "las direcciones en las que formulan su propia práctica de enseñanza" (p.282).

- En un tercer momento, se puede "observar y explicar el comportamiento de otros profesores y administradores en el sistema escolar" (p.282).

- En una cuarta etapa se puede, a manera de experimento, utilizar "nuevos enfoques del aprendizaje y de la enseñanza" (p.282).

En estudios empresariales un prácticum para podría centrarse en el método de casos. Éste se desarrollaría en varias direcciones:

1. Casos de "atención operativa": los estudiantes analizan la situación, expresan lo que otros harían y lo que ellos mismos harían. 
2. Casos de aplicación de principios: los estudiantes usan el caso como un "contexto" en el que descubren cómo se analizan los distintos elementos para saber si es prudente "llevar a cabo una operación" (Schön, 1987, p.282). De esta manera se ayuda al estudiante a razonar para determinar qué "temas y principios" deben tenerse en cuenta en el análisis de un caso específico.

3. Casos de "experimento": se lleva a los estudiantes más lejos, sobre todo cuando los principios aprendidos no son suficientes para solucionar un problema. Realizan un experimento que consiste en encontrar una nueva manera, "clarificadora" de 'plantear el problema y de establecer una estrategia que sirva para comprobar si el problema se puede resolver en sus "limitaciones reales". Además se deja la posibilidad de volver a plantear el problema en caso de algo inesperado (p.282).

4. Se puede añadir a las direcciones anteriores el caso de teorías tácitas: aquí se trata de descubrir las "teorías tácitas subyacentes" que los futuros profesionales aportan en el planteamiento y la solución de un problema (p.283). Los estudiantes son motivados para que planteen las acciones que llevarían a cabo; luego se les invitaría a que explicitaran los valores, estrategias y modelos que subyacen a los esquemas de acción propuestos. Así precisarían cuáles teorías hay en su práctica y dispondrían de alternativas teóricas para la solución de nuevos problemas ${ }^{50}$.

50 "Un profesor de caso podría implicarles en un proceso de estas características preguntándoles, en primer lugar, como de costumbre: 'iqué harías?' Después podría reunir y comparar un conjunto de aquellos esquemas de acción propuestos e invitar a los estudiantes a intentar construir los valores, las estrategias de acción y los modelos subyacentes de los fenómenos que hacen que tales propuestas de acción parezcan aceptables a sus defensores. Cuando se implica en este tipo de tarea a empresarios
Estos tipos de casos pueden emplearse también en otras "disciplinas como teoría de las organizaciones, psicología social, psicología de la motivación o teorías sobre el comportamiento interno o externo del mercado" (p.283). De esta manera van a mirar de un modo diferente las teorías existentes "a medida que se dan cuenta de que poseen sus propias teorías tácitas comparables a éstas" (Ib.).

La enseñanza de casos puede ampliarse hasta incluir la reflexión sobre la práctica. Para el estudiante la pregunta no es sólo: '¿Qué harías en esta situación?' Sino '¿Mediante qué tipo de intervenciones tratarías de poner en práctica las acciones que resultarían eficaces para este contexto?'" (lb.).

Un prácticum para ingenierías podría centrarse en "proyectos de diseño simulados" (Schön, 1987, p.283). Puede tomar tres direcciones:

1. Diseño en proyectos individuales: los estudiantes podrían reflexionar y describir "sus vías de aproximación a las tareas del diseño" (lb.). Podrían considerar "otras formas de diseñar", como el arquitectónico, por ejemplo.

2. Teorías tácitas en proyectos en grupo: se podría reflexionarse sobre "los procesos del grupo, la división del trabajo y las formas de tomar decisiones" (lb.). Cuando los estudiantes tengan conciencia "de sus propias teorías tácitas sobre el diseño y sobre la actuación en grupo", se puede reflexionar sobre

en activo, su exposición a las teorías múltiples de la acción frecuentemente les hace conscientes de hasta qué punto su propia práctica está empapada de teoría; lo que sugiere sorprendentes posibilidades de disponer de teorías de la acción alternativas a las que ya poseen, y crea un interés por el problema de verificar, sintetizar o elegir entre opciones teóricas igualmente plausibles" (Schön, 1987, p.283). 
las "teorías del diseño y sobre el funcionamiento del grupo" (Ib.).

3. Marcos y valores en los proyectos individuales o grupales: "se podría prestar una atención especial a las situaciones de formulación del diseño" (lb.). Los estudiantes impondrán "a la situación sus propios límites e imágenes de coherencia" (p.284). Reflexionarán sobre los conflictos entre los distintos valores implicados en un diseño. Estos son "valores profesionales, organizativos y sociales" (Ib.). Una ayuda en esta reflexión sería recurrir a la consideración de "ejemplos tomados de la literatura y de la crítica literaria o de la historia y la filosofía". Así, en la medida en que sean conscientes de las contradicciones entre "marcos conceptuales" y entre los "diferentes sistemas de valores" (Ib.), comprenderán que los estudios humanistas enseñan cómo las personas resuelven sus dilemas para resolver sus conflictos axiológicos y teóricos (lb.).

El profesor Schön insiste en que la reflexión no la hace solamente el estudiante, sino que también deben hacerla los profesores y los tutores51. Estas reflexiones son vitales para la realizacíón del prácticum reflexivo (p.284).

El hecho de realizar un practicum no garantiza que todos los estudiantes lleguen siempre a un aprendizaje extraordinario. Por el contrario, éste se sitúa en algún punto de los ejes entre los siguientes polos:

- "Vocabulario en circuito cerrado / comprensión sustantiva". Emplea

51 "Los prácticos tendrán que aprender a reflexionar sobre sus propias teorías tácitas; los profesores de las disciplinas a hacerlo sobre los métodos de indagación implícitos en su propia práctica de investigación; los tutores a hacerlo sobre las teorías y los procesos que tienen que ver con su propia reflexionen la acción" (Schön, 1987, p.284). palabras sin referirlas a la práctica, o las refiere a los procesos realizados.

- "Procedimientos parciales / comprensión integral". Utiliza procedimientos sin referirlos a todo el diseño, o los integra en la red de un todo coherente.

- "Estrecho y superficial / ancho y profundo". Es capaz de resolver este problema concreto y aislado, o es hábil para resolver un problema con proyección a situaciones distintas.

- "Sobreaprendizaje / representaciones múltiples". Presta atención al método del tutor y lo repite devotamente, o lo considera como un método que puede ser criticado $y$ transformado.

La diferencia en los resultados depende fundamentalmente del trabajo del estudiante para integrar lo aprendido a sus modos de pensar y de actuar y también de la manera como dialoga con su tutor hasta llegar a una reflexión mutua en la acción ${ }^{52}$.

El investigador Ángel I. Pérez, con J. Gimeno Sacristán, en un libro denominado Comprender y transformar la enseñanza, escribe que el enfoque reflexivo sobre la práctica profesional de los profesores comienza con Dewey. Éste propone el famoso principio de aprender haciendo, learning by doing, “y su no menos influyente propuesta de formar un profesor/a reflexivo que combine las capacidades de búsqueda e investigación con las actitudes de apertura mental, responsabilidad y honestidad" (Sacristán y Pérez, 1992, p.413) ${ }^{53}$.

52 "El lugar en que desemboque el aprendizaje de un estudiante, a lo largo de estas secuencias, depende de cómo él traslade los mensajes del prácticum a sus propias apreciaciones y a su propia ejecución, lo que, a su vez, depende de cómo transcurra el diálogo con su tutor. En la medida en que ese diálogo se acerque al ideal de la mutua reflexión en la acción (...) el aprendizaje del estudiante tiende a ser más amplio y profundo y, también, más sustantivo, integral y múltiple" (Schön, 1987, p.155).

53 Para John Dewey, la reflexión es el discernimiento entre la acción 
El mismo Ángel Pérez considera a Schön como el descubridor de "una nueva epistemología de la práctica profesional" (Sacristán y Pérez, 1992, p.416). Distingue en el denominado pensamiento práctico, tres niveles conceptuales diferentes"

1. Conocimiento en la acción,

2. Reflexión en la acción, y

3. Reflexión sobre la acción y sobre la reflexión en la acción (lb.).

Conocimiento en la acción: es un conocimiento de primer orden, "un componente inteligente que orienta toda actividad humana" (Sacristán y Pérez, 1992, p.418). Es un saber hacer que se halla presente en mi acción actual como resultado de una gran cantidad de experiencias y de reflexiones pasadas, "consolidado en esquemas semiautomáticos o rutinas" (Ib.). Es un conocimiento tácito, que por lo general es muy difícil de verbalizar ${ }^{54}$. Se

y su efecto, y está presente en toda experiencia significativa del hombre hasta el punto que llega a asimilarla con el pensamiento: “El pensamiento o la reflexión, como ya hemos visto virtual si no explícitamente, es el discernimiento de la relación que existe entre lo que tratamos de hacer y lo que ocurre como consecuencia. Ninguna experiencia con sentido es posible sin algún elemento de pensamiento" (Dewey, 1997, p. 128). Sin embargo, la reflexión sobre una experiencia puede ser "grosera", poca, o "de excelencia", mucha, o no darse, sencillamente: "podemos oponer dos tipos de experiencia según la proporción de reflexión que se encuentra en ellas" (p. 128). Cuando la reflexión es poca, o grosera, la experiencia se da con el método denominado de "ensayo y error". "Vemos que un cierto modo de actuar y una cierta consecuencia están conexionados, pero no vemos cómo lo están" (p. 128). Cuando la reflexión es mucha, o de excelencia, se procede de otra manera: "Analizamos para ver justamente lo que se halla en medio para ligar la causa y el efecto, la actividad y la consecuencia" (p. 128). Este tipo de reflexión hace ver la "continuidad" entre la acción y sus consecuencias, "hace posible actuar con un fin a la vista" (p. 129), y establece la responsabilidad de las acciones del sujeto humano.

Es posible que no haya reflexión sobre una experiencia y entonces se tienen la "rutina" y la "conducta caprichosa". La acción rutinaria "acepta lo acostumbrado como una plena medida de posibilidad y deja de tener en cuenta las conexiones de las cosas particulares hechas" (p. 129). La conducta caprichosa "hace del acto momentáneo una medida de valor e ignora las conexiones de nuestra acción personal con las energías del ambiente" (p. 129). Pero lo más grave es que una y otra "se niegan a reconocer la responsabilidad por las futuras consecuencias que se desprenden de la acción presente" (p. 129).

54 “Toda acción competente, incluso espontánea o improvisada, encuentra en muchas situaciones prácticas, "en la utilización de instrumentos o artefactos, en el reconocimiento de personas, objetos, procedimientos" (lb.). Pérez relaciona este conocimiento con el "conocimiento técnico o solución de problemas según Habermas" (Ib.).

Reflexión en o durante la acción: se presenta encima del conocimiento tácito en el mismo momento en que actuamos. Es un conocimiento de segundo orden, un meta-conocimiento en la acción. Es "un proceso de diálogo con la situación problemática y sobre la interacción particular que supone la intervención en ella" (Sacristán y Pérez, 1992, p.418). Esta reflexión se da en vivo, en caliente, sobre la actuación misma, y por eso no posee "la parsimonia, sistematicidad y distanciamiento que requiere el análisis racional", al tiempo que se impregna de "componentes emotivos o pasionales que condicionan la actuación y su reflexión" (lb.). Pero su importancia es grande ya que es el "primer espacio de confrontación empírica de los esquemas teóricos y de las creencias implícitas" en la acción, con las teorías y modelos existentes, y con la realidad misma. Gracias a ella se pueden confirmar o refutar los primeros, mediante re direccionamientos de la acción profesional. Por esto es "el mejor instrumento de aprendizaje significativo" (p.419) ${ }^{55}$. Pérez ve equivalente esta reflexión a la "deliberación práctica para Habermas" (lb.). revela un conocimiento normalmente superior a la verbalización que puede hacerse del mismo. Saber hacer y saber explicar lo que uno hace y el conocimiento y las capacidades que utiliza cuando actúa de forma competente, son en realidad dos capacidades intelectuales distintas" (Sacristán y Pérez, 1992, p.418).

55 "No sólo se aprenden y construyen nuevas teorías, esquemas y conceptos, sino que, lo que es más importante a nuestro entender, se aprende también el mismo proceso dialéctico de aprendizaje en 'conversación abierta con la situación práctica'" (Sacristán y Pérez, 1992, p.419). 
Reflexión sobre la acción y sobre la reflexión en la acción: se produce después de haber actuado, a posteriori, sobre la representación o reconstrucción de la propia acción. Es un conocimiento de tercer orden que "analiza el conocimiento en la acción y la reflexión en la acción en relación con la situación problemática y su contexto" (Sacristán y Pérez, 1992, p.420). Posee una ventaja: que se hace "en forma reposada y sistemática"; pero tiene el inconveniente de que se trabaja con un recuerdo, cuya objetividad debe estar validada por otros medios diferentes a la memoria (p.419). “La reflexión sobre la acción es un componente esencial del proceso de aprendizaje permanente que constituye la formación del profesional" (Ib.)56. Es un magnífico recurso para cuestionar todo lo actuado y lo implícito en la acción, y para establecer nuevos puntos de partida prácticos y teóricos. Ángel Pérez compara esta reflexión con la "reflexión crítica según Habermas" (lb.).

El investigador Pérez analiza ahora la reflexión desde Grimmett en su comentario sobre la reflexión de Schön. Grimmet expone tres modalidades de reflexión:

a. “Reflexión como acción mediatizada instrumentalmente" (Sacristán y Pérez, p.421). Se piensa sobre qué acciones recomendadas por la investigación empírica son más eficaces57.

56 "En dicho proceso se abre a consideración y cuestionamiento individual o colectivo no sólo las características de la situación problemática sobre la que actúa el práctico sino los procedimientos utilizados en la fase de diagnóstico y definición del problema, la determinación de metas, la elección de medios y la propia intervención que desarrolla aquellas decisiones; y, lo que en nuestra opinión es más importante, los esquemas de pensamiento, las teorías implícitas, creencias y formas de representar la realidad que utiliza el profesional en las situaciones problemáticas, inciertas y conflictivas" (Sacristán y Pérez, 1992, p.420).

57 "La reflexión en esta perspectiva cumple una función instrumental y clarificadora con la pretensión de entender mejor las propuestas y métodos de intervención elaborados por especialistas externos, la fuente de conocimiento es generalmente una autoridad exterior y, parece claro, que el conocimiento teórico dirige la práctica" b. “Reflexión como proceso de deliberación entre diversas y a menudo enfrentadas orientaciones" (Ib.). Se considera el contexto y se evalúan las consecuencias al adoptar cada una de las diferentes orientaciones $^{58}$.

c. "Reflexión como reconstrucción de la experiencia" (lb.). Se rehace la propia experiencia desde tres instancias paralelas ${ }^{59}$ :

a. "Reconstruir las situaciones donde se produce la acción" (lb.). Se redefine la situación problemática teniendo en cuenta circunstancias y características no tenidas en cuenta, o reinterpretadas de nuevo.

b. "Reconstruirse a sí mismos" (Ib.) como profesionales. Se reconsidera la forma propia de estructurar sus "conocimientos, sus afectos y sus estrategias" (Ib.).

c. "Reconstruir los supuestos(...) aceptados como básicos" (Ib.). Se analizan críticamente las razones y los intereses que dependen de los principios y formas de concebir la profesión.

Brockbank y McGill consideran, en su propuesta pedagógica, el término reflexión en doble sentido. "Lo utilizamos en dos sentidos. En primer lugar, como el procedimiento o medio por el que se considera una experiencia, en forma de pensamiento, sentimiento o acción, mientras está ocurriendo o posterior-

(Sacristán y Pérez, p.421).

58 “También aquí la base de conocimiento es la autoridad externa pero el modo de conocer ya es deliberativo, a través de contraste con los colegas y en función de las necesidades del contexto. El conocimiento, que se considera en cierta medida siempre relativo, ya no dirige la acción sino simplemente la informa, orienta" (lb.).

59 "Este último modo de concebir la reflexión va, a nuestro entender, más allá del planteamiento de Schön, aunque se apoya fuertemente en sus aportaciones sobre la reflexión en la acción, al plantear la reconstrucción dialéctica del quehacer (...) penetrando en las condiciones políticas, sociales y económicas que afectan su pensamiento y su acción, así como el escenario donde se interviene" (Sacristán y Pérez, p.422). 
mente. El segundo sentido, derivado del primero, es la creación de significado y la conceptualización partiendo de la experiencia y la potencialidad de mirar las cosas como diferentes de lo que son" (Brockbank y McGill, 2002, p. 72). Estos tipos de reflexión aparecen más claros después de considerar las dimensiones del proceso reflexivo.

Brockbank y McGill proponen, a partir de los trabajos de Schön, cinco dimensiones en el proceso de reflexión en la acción y sobre la acción. Éstas son:

\section{Acción}

2. Reflexión-en-la-acción

3. Descripción de la reflexión-en-laacción

4. Reflexión sobre la descripción de la reflexión-en-la-acción (reflexión sobre la acción)

5. Reflexión sobre la reflexión sobre la acción" (p. 98).

Habría, entonces, tres niveles de reflexión: la reflexión-en-la-acción; la reflexión-sobre-la-acción; y la reflexión sobre la reflexión-sobre-la-acción. La primera se da mientras se está realizando la acción ${ }^{60}$. La segunda, después de ocurrida la acción y es un momento de relativa libertad y profundidad en la reflexión. La tercera es una instancia reflexiva eminentemente crítica ${ }^{61}$.

60 “La reflexión-en-la-acción se produce cuando nos encontramos en medio de una acción y, al hacerla y mostrarnos reflexivos-en-la-acción, decimos, por ejemplo:

- está ocurriendo algo que me sorprende; no es habitual;

- ¿es adecuado en este momento lo que estoy haciendo?..

Para Schön, lo que distingue la reflexión-en-la-acción de otras formas de reflexión es 'su significado inmediato para la acción (...) el replanteamiento de alguna parte de nuestro saber-en-la-acción lleva a un experimento sobre la marcha y a nuevos pensamientos que influyen en lo que hagamos -en la situación en la que nos encontremos y quizá también en otras que nos parezcan similares a ella-'(Schön, 1987, pág.29)" (Brockbank y McGill, p.90).

61 "En esta dimensión, los alumnos trabajan sobre la significación de la misma reflexión, es decir: iAprenden a aprender! Por tanto,
Brockbank y McGill, en su propuesta pedagógica, sugieren que antes de empezar a practicar la reflexión desde la acción con los estudiantes, el profesor universitario practique estas aplicaciones, una después de otra:

I. Reflexión personal

II. Diálogo reflexivo con otro colega: contar la historia

III. Diálogo reflexivo con otro colega: formar parte de la historia

IV. Diálogo reflexivo con otros colegas: facilitar el desarrollo de la práctica reflexiva" (Brockbank y McGill, p.128)62.

Cuando ha realizado lo anterior, el profesor comenzará la práctica de la reflexión desde la acción con sus estudiantes en las aulas de clase. Para ello organiza el grupo en subgrupos de a tres estudiantes. Estos tres tienen cada uno un rol distinto: el de presentador, el de capacitador y el de informador. En este momento puede llevar a cabo el seminario reflexivo en tres partes, que los autores describen de esta manera:

Al principio de la primera parte, el profesor, en calidad de facilitador, presenta el diálogo reflexivo al grupo completo de alumnos, modelando el procedimiento y orientándoles para que inicien la fase de información inicial para discutir y planear un ensayo que deben realizar todos los integrantes del grupo. El profesor tiene que informar de antemano a

en el diálogo reflexivo sobre esta dimensión, el individuo puede aprender gracias a la interacción en el grupo" (p.97).

62 "Las aplicaciones parten de la reflexión personal 'a solas' (Aplicación I) y atraviesan diversos niveles de participación con otros profesionales compañeros en el diálogo. En la Aplicación II, un docente reflexiona con un colega después de desarrollar un acto de práctica profesional. En la Aplicación III, un profesor reflexiona con un colega antes, 'durante' y después de un acto docente desarrollado ante el compañero. La Aplicación IV presenta a un docente o facilitador que promueve el desarrollo de la práctica reflexiva con un grupo de compañeros con el formato de un seminario" (p.128). Este seminario es parecido al seminario de los estudiantes que después será descrito. 
los estudiantes de los requisitos que deben cumplir, p. Ej., el tema del ensayo y los criterios de evaluación. En esta fase de información inicial, los estudiantes se reparten en tríadas para planear el enfoque del ensayo que utilice cada uno, en relación con los objetivos, contenidos y el procedimiento. Por turno, cada alumno de la tríada decide su enfoque de la preparación del ensayo. Al terminar esta fase, comienzan a preparar el borrador, basándose en las intenciones determinadas en la primera etapa. Después de acabar el borrador, vuelven al seminario para celebrar la segunda fase (segunda parte), formando las mismas tríadas, para revisar los borradores. Lo ideal sería que cada uno hubiera presentado con antelación el borrador a sus compañeros y que éstos lo hubieran leído. En caso contrario, el borrador se presenta en el seminario. Finalizada la segunda parte, cada alumno completa la tarea, el ensayo, y lo entrega al profesor responsable de su evaluación y calificación.

Tras la devolución de los ensayos evaluados y calificados (con los comentarios pertinentes del tutor), los estudiantes se reúnen en la tercera fase (tercera parte) del seminario, para informar sobre lo acontecido. La finalidad de la sesión de información posterior es que cada alumno articule lo aprendido en la realización de la tarea y acerca de su enfoque del aprendizaje. Esta sesión no está pensada como última oportunidad para cuestionar o discutir las calificaciones otorgadas por el tutor (Brockbank y McGill, p.152).

El estudiante con el rol de presentador llevará a cabo la tarea o la práctica requerida ${ }^{63}$. El que tiene el rol de ca-

63 “El estudiante que actúa como presentador desempeña un papel real. Se prepara para redactar un ensayo, pero con el apoyo y las cuestiones que suscite otro compañero en esta fase de pacitador le colaborará al presentador en la reflexión durante el curso de su práctica ${ }^{64}$. El que desempeña el rol de informador anotará los diálogos y las acciones de los otros dos compañeros65.

Sin demeritar la propuesta anterior, el doctor Schön pretende que la reflexión desde la acción vaya más allá del aula, se integre a las prácticas profesionales y contribuya en la elaboración de los curricula de los pregrados y los posgrados.

Donald Schön, formando un equipo con cinco docentes y dos estudiantes, realizó la reforma del Currículum del posgrado denominado Master sobre Planificación Urbanística del MIT. EI currículum vigente consideraba cuatro materias fundamentales: economía, métodos cuantitativos, proceso de planificación y análisis institucional. El equipo de Schön hizo algunos cambios en las asignaturas, transformando la Economía en Economía política para planificadores, los métodos cuantitativos en razonamieno cuantitativo, y las otras dos en Procesos institucionales y de planificación (Schön, 1987, p.289). La

información inicial, que también dialogará con él sobre el borrador del trabajo en la fase siguiente (segunda parte). El hecho de pensar en el ensayo propuesto tiene la ventaja de articular sus intenciones y de plantear los problemas y preocupaciones potenciales antes de redactar el borrador. Está desarrollando la primera parte de la práctica reflexiva" (Brockbank y McGill, p.157).

64 “El compañero también desempeña un papel real, porque participa en la sesión de retroinformación al capacitador sobre el grado en que ha cumplido su finalidad. Además, el informador también puede proporcionar retroinformación al presentador, en relación con su forma de responder al capacitador, así como comunicarle cómo 've' las cuestiones tal como se hayan presentado, p.ej., en torno al compromiso (...)" (p.157).

65 El compañero que actúa como informador desempeña un papel fundamental. Como informador, toma nota, en primer lugar, de las aportaciones del capacitador al diálogo de la sesión de información inicial. Su finalidad principal consiste en dar fe y registrar hasta qué punto faculta verdaderamente el capacitador al presentador para que utilice sus pensamientos, sentimientos y acciones potenciales en relación con el ensayo previsto. Concluido el diálogo, el informador facilita retroinformación al capacitador sobre el grado en que ha cumplido su finalidad. Además, el informador también puede proporcionar retroinformación al presentador, en relación con su forma de responder al capacitador, así como comunicarle cómo 've' las cuestiones tal como se hayan presentado, p.ej., en torno al compromiso (...)" (p.157). 
planificación de estas asignaturas facilitaba su interrelación y la interacción con un proyecto integrador que consideraba los problemas de una zona de Boston ubicada entre Copley Square y Blue Hill Avenue. El nuevo currículum fue sustentado en 1983 para la evaluación de profesores y alumnos ${ }^{66}$.

El proyecto en su totalidad constituía el prácticum reflexivo, y en él los estudiantes llevaban a cabo la reflexión desde la práctica en todos sus aspectos y la integración con las asignaturas planificadas ${ }^{67}$. La evaluación de este prácticum fue expresada concisamente por el mismo Schön: "Algunas de nuestras pretensiones se vieron totalmente satisfechas; otras, sólo de manera parcial o de ninguna manera" (Schön, 1987, p.291).

\section{REFERENCIAS}

BÁRCENA, Fernando y MÈLICH, Joan-Carles (2000), La educación como acontecimiento ético. Barcelona: Paidós.

BERGER, Peter L. y LUCKMANN, Thomas (), La construcción social de la realidad, $11^{a}$ reimpresión. Buenos Aires: Amorrortu Editores, 1993.

BLUMER, Herbert (1969), Symbolic interactionism. Perspective and Method

FIRST PAPERBACK PRINTING. NEW JERSEY: PRENTICEHALL, 1986.

BROCKBANK, Anne y McGILL, lan, Aprendizaje reflexivo en la educación superior. Madrid: Morata, 2002.

DEWEY, John, Democracia y educación (), $2^{\mathrm{a}}$ ed. Madrid: Morata, 1997,

FERNÁNDEZ CHRISTLIEB, Pablo (1994), La psicología colectiva un fin de siglo más tarde. Su disciplina. Su conocimiento. Su realidad. Santafé de Bogotá: Anthropos.

66 "Habíamos intentado diseñar nuestros tres cursos de forma tal que sus bases conceptuales resultasen mucho más claras de lo que habían sido anteriormente, $y$, además, se pudiesen conectar mejor entre sí y, a la vez, con el proyecto Copley Place / Blue Hill Avenue. Como resultado, en parte, de este esfuerzo de integración, habíamos acordado asistir unos a las clases de los otros" (Schön, 1987, p.290-291).

67 “Queríamos que nuestro prácticum, a modo de taller, cumpliese distintas funciones. Queríamos utilizarlo como una especie de prisma a través del cual pudiéramos ver la utilidad de las ideas y los métodos utilizados en los cursos a que hice referencia anteriormente. Y deseábamos que fuera, además, un instrumento para que estudiantes y profesores reflexionasen sobre el proceso de la formulación de problemas en aquellas situaciones confusas y conflictivas extraídas de la práctica real de la planificación, un marco en el que los alumnos reflexionarían acerca de las teorías tácitas que ellos aportaban a las soluciones de su proyecto y en el que pondrían a prueba los métodos recién adquiridos de análisis y descripción cuantitativos" (p.291)
FOUREZ, Gérard, La construcción del conocimiento científico. Madrid: Narcea, 1998.

MEAD, George Herbert (1927: 1934, póstumo), Espíritu, persona y sociedad. Desde el punto de vista del conductismo social. Buenos Aires: Paidós, 1972, en FERNANDEZ CHRISTLIEB, Pablo, La psicología colectiva un fin de siglo más tarde. Su disciplina. Su conocimiento. Su realidad. Santafé de Bogotá: Anthropos, 1994, ps. 70 a 85.

MÈLICH, Joan-Carles (1994), Del extraño al cómplice. La educación en la vida cotidiana. Barcelona: Anthropos.

MORIN, Edgar (1999), La mente bien ordenada, $2^{\mathrm{a}}$ ed. Barcelona: Seix Barral, 2001.

NOT, Louis (1979), Las pedagogías del conocimiento, $3^{\mathrm{a}}$ reimp. en español. Santafé de Bogotá: Fondo de Cultura Económica, 1998.

NOT, Louis (1989), La enseñanza dialogante. Barcelona: Herder, 1992.

NOVAK, Joseph D. (1977), Teoría y práctica de la educación. Madrid: Alianza Editorial, 1982.

SACRISTÁN, J. Gimeno y PÉREZ GÓMEZ, A. I. (1992), Comprender y transformar la enseñanza, $3^{\mathrm{a}}$. ed. Madrid, Morata, 1994

SCHÖN, Donald A. (1983), El profesional reflexivo. Cómo piensan los profesionales cuando actúan. Barcelona: Paidós, 1998.

SCHÖN, Donald A. (1987), La formación de profesionales reflexivos. Hacia un nuevo diseño de la enseñanza y el aprendizaje en las profesiones. Barcelona: Paidós, 2002.

SCHÜTZ, Alfred (1932), La construcción significativa del mundo social. Introd 